\title{
IMPACTO DE LA COVID-19 EN EMPRESAS AGROALIMENTARIAS EXTREMEÑAS: ESTUDIO DE CASOS MÚLTIPLE
}

\author{
Beatriz Corchuelo ${ }^{a *}$, Pedro Eugenio López ${ }^{\mathrm{b}}$, Celia Sama $^{\mathrm{a}}$. \\ ${ }^{a}$ Universidad de Extremadura. Facultad de Ciencias Económicas y Empresariales. Departamento de \\ Economía (Badajoz,bcorchue@unex.es y celiasamaberrocal@unex.es). \\ ${ }^{b}$ Universidad de Extremadura. Facultad de Ciencias Económicas y Empresariales. Departamento de \\ Dirección de Empresas y Sociología (Badajoz, pelopez@unex.es).
}

\begin{abstract}
Resumen
La crisis producida por la COVID-19 ha alterado las pautas de producción y consumo de alimentos a nivel mundial. El objetivo de este estudio es analizar el impacto que ha tenido en el sector agroalimentario en Extremadura. Los resultados informan de que el cambio en las condiciones del entorno y las características de la difusión de la pandemia han tenido un efecto en las estrategias, el comportamiento, los procesos, la dinámica y los resultados de las organizaciones, independientemente de su tamaño y la naturaleza de su producto o servicio.
\end{abstract}

Palabras clave: Covid-19, empresas agroalimentarias, estudio de casos, impacto, actuaciones.

\section{Introducción y objetivos}

La pandemia de coronavirus está teniendo un impacto económico no existente previamente en la economía mundial. Según Gjaja et al. (2020) se trata de una crisis de escala y complejidad histórica que está poniendo a prueba los sistemas de atención médica, la capacidad fiscal de los gobiernos y la capacidad de muchas organizaciones para hacer frente a los cambios provocados por el virus y de darle respuesta a los mismos.

En España, las estrictas medidas de confinamiento vigentes en 2020, así como las restricciones sobre el turismo internacional, han supuesto una caída histórica del producto interior bruto (PIB), lo cual ha afectado al sector agroalimentario [CaixaBank (2020)].

El sector agroalimentario agrupa un conjunto de actividades muy heterogéneas y en él interactúan múltiples variables que influyen directamente sobre el bienestar de la sociedad en su conjunto, lo que hace que en la actualidad se enfrente a un reto no contemplado previamente de nuevos patrones de consumo y de percepciones e inquietudes de los consumidores. Sin embargo, en España, este sector ha sido uno de los menos afectados por la crisis de la COVID-19. En Extremadura, el peso del sector agrario y sus industrias asociadas sustancialmente superiores a la media nacional.

En este marco, el objetivo de este estudio es analizar el impacto de la COVID-19 en las empresas agroalimentarias extremeñas. El análisis se realiza a través de las apreciaciones de responsables en gestión de 15 empresas agroalimentarias pertenecientes a diferentes ramas de actividad y tamaño, y del director de un centro tecnológico agroalimentario con gran peso en la región. Este estudio pretende contribuir a la aún escasa literatura existente sobre el impacto de la pandemia en el sector agroalimentario [Barcaccia et al. (2020); Patterson et al. (2020); Montanari et al. (2020); Scorza et al. (2020)], en este caso referido a una región concreta.

\section{Metodología}

Se utiliza una metodología cualitativa a partir de un estudio de casos múltiples y se adopta un enfoque exploratorio. De acuerdo con Yin (1989) y Tell et al. (2016), esta metodología es adecuada a los objetivos del estudio.

Una actividad previa, fue la elaboración de un informe/directorio de las empresas agroalimentarias de Extremadura a través del cruce y análisis de diferentes bases de datos (Instituto Nacional de Estadística, Cooperativas Agroalimentarias Extremeñas y SABI). El informe contiene datos de 734 empresas agroalimentarias de diversas formas jurídicas y tamaños y permitió seleccionar las empresas en base a diversos criterios, como la pertenencia a ramas de actividad que desarrollan o producen productos altamente especializados en la región, diferentes tamaños y formas legales (por ejemplo, cooperativas agroalimentarias) y si son organizaciones dinámicas e innovadoras. Después se realizaron las entrevistas en profundidad a responsables de gestión de 15 empresas agroalimentarias extremeñas y un centro tecnológico con importante peso en la región. Las cuestiones formuladas en el guión de la entrevista 
estuvieron relacionadas con el impacto de la COVID-19 (efecto negativo y positivo), sí como las acciones y cambios que habían llevado a cabo las organizaciones en sus actividades. Por ejemplo: ¿Consecuencias de la pandemia?, ¿Consecuencias con efecto positivo?, ¿Qué acciones se están llevando a cabo?

La Tabla 1 se muestra las características de las entrevistas. La numeración de las empresas corresponde con el orden de las entrevistas.

Tabla 1. Características de las organizaciones entrevistadas

\begin{tabular}{|c|c|c|c|c|c|c|}
\hline Empresa & $\begin{array}{c}\text { Actividad } \\
\text { CNAE2009 }\end{array}$ & $\begin{array}{c}\mathrm{N}^{\mathrm{o}} \\
\text { empleados }\end{array}$ & Facturación & Exporta & $\begin{array}{c}\text { Persona } \\
\text { entrevistada }\end{array}$ & $\begin{array}{l}\text { Fecha } \\
\text { entrevista }\end{array}$ \\
\hline Empresa 1 & 103 & 600 & $\begin{array}{c}> \\
10,000,000 €\end{array}$ & $\mathrm{Si}$ & $\mathrm{CFO}$ & $28 / 02 / 2020$ \\
\hline Empresa 2 & 104 & 3 & $\begin{array}{l}1,000,001- \\
2,000,000 €\end{array}$ & $\mathrm{Si}$ & CEO & $13 / 03 / 2020$ \\
\hline Empresa 3 & 110 & 3 & $0-500,000 €$ & Sí & $\begin{array}{l}\text { Directora } \\
\text { comercial }\end{array}$ & 08/06/2020 \\
\hline Empresa 4 & 110 & 10 & $\begin{array}{l}1,000,001- \\
2,000,000 €\end{array}$ & Sí & CEO & $18 / 06 / 2020$ \\
\hline Empresa 5 & 108 & 1 & $0-500,000 €$ & No & CEO & $25 / 06 / 2020$ \\
\hline Empresa 6 & 109 & 70 & $>10,000,000 €$ & Sí & CEO & 06/07/2020 \\
\hline Empresa 7 & 103 & 60 & $>10,000,000 €$ & Sí & $\begin{array}{l}\text { Director de } \\
\text { operaciones }\end{array}$ & 07/07/2020 \\
\hline Empresa 8 & 104 & 35 & $>10,000,000 €$ & Sí & CEO & 08/07/2020 \\
\hline Empresa 9 & 101 & 9 & $\begin{array}{c}500,001- \\
1,000,000 €\end{array}$ & Sí & CEO & $18 / 07 / 2020$ \\
\hline Empresa 10 & 109 & 10 & $\begin{array}{c}6,000,001- \\
10,000,000 €\end{array}$ & Sí & $\begin{array}{c}\text { Responsable } \\
\text { de } \\
\text { departamento }\end{array}$ & $20 / 07 / 2020$ \\
\hline \multirow{2}{*}{ Empresa 11} & \multirow{2}{*}{101} & \multirow{2}{*}{66} & \multirow{2}{*}{$>10,000,000 €$} & \multirow{2}{*}{ Sí } & $\begin{array}{l}\text { Director de } \\
\text { operaciones }\end{array}$ & $28 / 09 / 2020$ \\
\hline & & & & & CEO & $06 / 10 / 2020$ \\
\hline Empresa 12 & 101 & $<200$ & $>10,000,000 €$ & Sí & $\begin{array}{c}\text { Responsable } \\
\text { de } \\
\text { departamento }\end{array}$ & 05/10/2020 \\
\hline Empresa 13 & 103 & 110 & $>10,000,000 €$ & Sí & CEO & $15 / 10 / 2020$ \\
\hline Empresa 14 & 103 & 55 & $>10,000,000 €$ & Sí & CEO & $30 / 10 / 2020$ \\
\hline Empresa 15 & 103 & 6 & $\begin{array}{c}6,000,001- \\
10,000,000 €\end{array}$ & Sí & CEO & $12 / 01 / 2020$ \\
\hline $\begin{array}{c}\text { Centro } \\
\text { Tecnológico }\end{array}$ & 721 & 49 & $>2,000,000 €$ & No & CEO & 07/10/2021 \\
\hline
\end{tabular}

Notas: 101: Procesado y conservación de carne y elaboración de productos cárnicos; 103: Procesado y conservación de frutas y hortalizas; 104: Fabricación de aceites y grasas vegetales y animales; 108: Fabricación de otros productos alimenticios; 109: Fabricación de productos para la alimentación animal; 110: Fabricación de bebidas; 721: Investigación y desarrollo experimental en ciencias naturales y técnicas.

Fuente: Elaboración propia. 
Es importante puntualizar que esta investigación procede de un estudio previo que examina la innovación en el sector agroalimentario en Extremadura [Corchuelo, et al. (2020)]. Por eso, ante las numerosas noticias que ya circulaban en los medios de comunicación debido a la pandemia y coincidiendo con el comienzo de las entrevistas en el estudio previo, surgió la oportunidad de incluir preguntas que permitiesen conocer y analizar cuál ha sido el impacto de la pandemia para las empresas agroalimentarias extremeñas. Por este motivo, la primera y segunda entrevista (Empresas 1 y 2) se realizaron de forma presencial en la sede de las empresas antes de que el Gobierno de España decretase el estado de alarma.

Por tamaños, solo una de las empresas tiene más de 200 trabajadores. El 33\% de las empresas son microempresas (tienen menos de 10 trabajadores). Cuatro de las empresas entrevistadas son cooperativas agroalimentarias (empresas 4, 6, 13 y 15). Excepto una empresa, todas las organizaciones declararon ser exportadoras. En cuanto a actividades (según CNAE2009), el 33,3\% corresponde a "Procesado y conservación de frutas y hortalizas", el 20\% a "Procesado y conservación de carne y productos cárnicos", el 13,3\% a los grupos de actividad "Fabricación de aceites vegetales y otras grasas animales", "Fabricación de bebidas" y "Fabricación de productos para alimentación animal", y un 6,7\% a "Fabricación de otros productos alimenticios".

\section{Resultados}

El análisis y codificación de las transcripciones de las entrevistas y otra información descriptiva secundaria se realizó a través del software informático WebQDA que permitió extraer los principales resultados sobre el impacto de la COVID-19 en empresas agroalimentarias extremeñas [Corchuelo et al. (2021)].

En general, como consecuencias negativas se observaron las siguientes: disminución de la facturación y ventas; reducción y desplazamiento en la demanda de productos; dificultades en actividades de comercialización; ralentización en el desarrollo de proyectos de $\mathrm{I}+\mathrm{D}+\mathrm{i}$; falta de personal (cuarentena), disminución de las actividades desarrolladas y problemas de abastecimiento por los proveedores. Las industrias agroalimentarias más perjudicadas y que más han disminuido la facturación son aquellas que dirigen la comercialización de sus productos al canal Horeca y productos asociados a celebraciones. Igualmente, los productos de mayor valor en el mercado también se han visto afectados por la inestabilidad económica ocasionando que descienda el consumo y se desplace la demanda hacia aquellos productos con similar posicionamiento y menor precio en el mercado. Sin embargo, las empresas menos afectadas son las que tienen diversificados los canales de venta.

Por otro lado, se observa que en las organizaciones que están dedicadas al procesado y conservación de frutas y hortalizas, la pandemia tuvo consecuencia con efecto positivo, ya que se produjo un aumento de la demanda de sus productos.

En base a las consecuencias ocasionadas desde que se decretase el estado de alarma en España, las empresas han acelerado y llevado a cabo una serie de actuaciones para paliar los efectos de la pandemia. Entre las principales actuaciones llevadas a cabo por las empresas: búsqueda y diversificación de clientes y proveedores en otros mercados; aumento de la gama de productos; reorganización de las actividades y el personal; introducción de nuevos protocolos higiénicos en los centros de trabajo para garantizar la seguridad y evitar contagios, así como el aumento del uso de la tecnología en sus actividades de comercialización (creación de páginas web y tiendas online) y comunicación (herramientas de comunicación a distancia).

\section{Conclusiones}

La crisis sanitaria provocada por el COVID-19 está teniendo importantes consecuencias en la economía en su conjunto, en los sectores y en las ramas de la actividad económica mundial. El análisis de las respuestas ha permitido conocer que la pandemia ha traído consigo principalmente consecuencias con efecto negativo. De forma particular, se han observado consecuencias con efecto positivo, como es el aumento de la demanda de sus productos en determinadas empresas que están produciendo y realizando la primera transformación de cereales y cultivos agrícolas.

Los resultados obtenidos revelan que, aunque la industria agroalimentaria pertenece a un sector imprescindible y estratégico en la economía extremeña, la pandemia también ha influido en este sector, aunque no con el mismo impacto que en otros.

En definitiva, y como principal conclusión, el cambio en las condiciones del entorno, junto con las características de propagación de la pandemia, han impactado en las estrategias, comportamientos, procesos, dinámicas y resultados de las empresas agroalimentarias extremeñas independientemente de su tamaño y la naturaleza de su entorno, producto o servicio. Para adaptarse a la nueva normalidad, pese a la destrucción generalizada de empleo y bajada de la productividad que ha causado la pandemia en otros sectores de actividad en España, las empresas agroalimentarias extremeñas están experimentando cambios 
en los modelos operativos y prioridades funcionales de sus negocios. Hay ciertos aspectos que, si bien ya se estaban adoptando medidas para ponerlos en práctica, se han acelerado, como la comunicación virtual, el teletrabajo y la digitalización, siendo este último aspecto fundamental para ganar competitividad.

\section{Bibliografía}

Barcaccia, G., D'Agostino, V., Zotti, A., \& Cozzi, B. (2020). Impact of the SARS-CoV-2 on the Italian Agri-food Sector: An Analysis of the Quarter of Pandemic Lockdown and Clues for a SocioEconomic and Territorial Restart. Preprints (www.preprints.org). Doi: 10.20944/preprints202007.095.v1.

CaixaBank Research (2020). Informe Sectorial Agroalimentario 2020: Resiliencia y crecimiento del sector durante la pandemia. https://www.caixabankresearch.com/es/agroalimentario/octubre2020/agroalimentario-resiliencia-y-crecimiento-del-sector-durante-pandemia

Corchuelo, B., López, P. E., \& Sama, C. (2020). Determining Factors of Innovative Performance: Case Studies in Extremaduran Agri-Food Companies. Sustainability, 12(21), 1-24. https://doi.org/10.3390/su12219098

Corchuelo, B., López, P. E., \& Sama, C. (2021). Impact of the COVID-19 Pandemic on Agri-Food Companies in the Region of Extremadura (Spain). Agronomy, 11(5), 971. https://doi.org/10.3390/agronomy11050971

Gjaja, M., Faeste, L., Hansell, G, \& Hohner, D. (2020). COVID 19: Win the Fight, Win the Future. www.BGC.com.https://www.bcg.com/publications/2020/covid-scenario-planning-winning-thefuture-series

Montanari, F., Arayess, S., Toma, B., Clavarino, A., Ferreira, I.M., Aude, M., Stelios, M. A., Schröck, C., Servé, A., Wesolowska, V., Velázquez, P. (2020). The Response of the EU Agri-Food Chain to the COVID-19 Pandemic:Chronicles from the EU and Selected Member States. European Food and Feed Law Review: EFFL; 15(4):336-356.

Patterson, G.T., Thomas, L.F., Coyne, L.A., Rushton, J. (2020) Moving health to the heart of agri-food policies; mitigating risk from our food systems. Global Food Security, 26. DOI: https://doi.org/10.1016/j.gfs.2020.100424

Scorza, F., Murgante, B., Pilogallo, A., Saganeiti, L., Santarsiero, V., Faruolo, G., Fortunato, G., Izzo, C.., Piro, R. y Bonifazi, A. (2020). Best Practices of Agro-Food Sector in Basilicata Region (Italy): Evidences from INNOVAGRO Project. International Symposium: New Metropolitan Perspectives (NMP 2020), 1706-1713.

Tell, J., Hoveskog, M., Ulvenblad, P., Ulvenblad, P-O., Barth, H. y Ståhl, J. (2016). Business model innovation in the agri-food sector: a literature review. British Food Journal, 118 (6), 1462-1476. DOI 10.1108/BFJ-08-2015-0293.

Yin, R. K. Case Study Research: Design and Methods (Applied Social ResearchMethods); Newbury Park CA, Sage, 1989. 